\title{
CONTAMINAÇÃo POR Toxocara spp. e Ancylostoma spp. EM ÁREAS DE LAZER DO MUNICÍPIO DE CANOINHAS, SC
}

\section{Contamination by Toxocara spp. and Ancylostoma spp. in areas of leisure from Canoinhas County, Santa Catarina state}

\author{
PEDRASSANI, D. ${ }^{1}$; VIERA, A.M. ${ }^{2}$; THIEM, E.M.B ${ }^{3}$
}

\footnotetext{
${ }^{1}$ Mestre em Ciências Veterinárias. Aluna do Curso de Pós-graduação em Medicina Veterinária - Medicina Veterinária Preventiva, FCAV - UNESP, Campus de Jaboticabal. Docente da Universidade do Contestado, UnC - Canoinhas, SC;

${ }^{2}$ Acadêmico do Curso de Medicina Veterinária da UnC - Canoinhas, SC;

${ }^{3}$ Auxiliar de laboratório da UnC - Canoinhas, SC.

Endereço para correspondência: Daniela Pedrassani - daniela@cni.unc.br
}

\section{RESUMO}

Larva migrans visceral e cutânea são zoonoses causadas por Toxocara spp. e Ancylostoma spp., respectivamente. O objetivo desta pesquisa foi avaliar a contaminação por estes agentes em áreas de lazer do município de Canoinhas, Santa Catarina, por meio das técnicas de sedimentação espontânea, centrífugo sedimentação e centrífugo flutuação. Das áreas de lazer examinadas $(n=5), 100 \%$ apresentaram a areia contaminada e 80\% apresentaram fezes contaminadas por Ancylostoma spp e/ou Toxocara spp. Destas, 100\% apresentaram contaminação pelo Ancylostoma spp e apenas uma apresentou contaminação com ovos de Toxocara spp.. A presença de Ancylostoma spp. e de Toxocara spp. nas amostras de areia analisadas foi de $39 \%$. Nos exames de fezes coletadas nas áreas de lazer foram detectadas $17 \%$ de amostras positivas, sendo detectado apenas ovos de Ancylostoma spp. O estudo revelou que a contaminação das áreas de lazer do município de Canoinhas é preocupante, reforçando a necessidade de implementar medidas efetivas para controle de zoonoses em áreas de lazer.

Palavras-chave: Ancylostoma; Toxocara, Larva migrans, saúde pública, zoonose.

\section{ABSTRACT}

Visceral and cutaneous larva migrans are zoonoses caused by the Toxocara spp. and Ancylostoma spp. respectively. The objective of this research was to evaluate the contamination for these agents in areas of leisure from the city of Canoinhas, Santa Catarina, by means of the techniques of spontaneous sedimentation, centrifugal sedimentation and centrifugal fluctuation. From the examined areas of leisure, $100 \%$ had disclosed the contaminated sand and $80 \%$ excrements contaminated by Ancylostoma spp and/or Toxocara spp.. Of these, $100 \%$ had presented contamination for the Ancylostoma spp and only one presented contamination with eggs of Toxocara spp..The presence of Ancylostoma spp. and Toxocara spp. in the analyzed sand samples was of $39 \%$. In the excrement examinations collected in the leisure areas $17 \%$ of positive samples had been detected, being detected only eggs of Ancylostoma spp. The study it demonstrated that the contamination of the areas of leisure of the Canoinhas city, is preoccupying, strengthening the necessity to implement measured effective for control of zoonoses in leisure areas.

Key words: Ancylostoma, Toxocara, Larva Migrans, public health, zoonose. 


\section{INTRODUÇÃO}

Os animais de companhia desempenham importante papel nas sociedades, pois contribuem para o desenvolvimento físico, social e emocional de crianças e para o bem estar de seus proprietários (Robertson et al., 2000). Entretanto, a vivência constante dos cães e gatos junto ao homem é uma possível causa de transmissão de agentes zoonóticos, e a permanência destes em locais públicos e até mesmo nas residências, pode ocasionar a contaminação do ambiente (Araújo et al., 1999). A contaminação ambiental por dejetos de animais domésticos que perambulam em vias públicas é motivo de preocupação tanto para os ambientalistas como para profissionais sanitaristas. As fezes de cães e gatos podem albergar uma série de parasitas de importância em saúde pública, como formas infectantes de Toxocara canis ou T. cati, e de Ancylostoma braziliense e $A$. caninum (Matesco et al., 2006).

A circulação desses animais em áreas públicas, principalmente em playgrounds, é importante devido à presença constante de crianças, ainda sem noções completas de higiene e saúde. Com isso, as canchas de areia dessas áreas de lazer representam risco potencial aos freqüentadores de contraírem zoonoses parasitárias, como a larva migrans cutânea (LMC) e a larva migrans visceral (LMV) (Santarém et al., 1998).

A LMC é uma dermatite causada pela migração de larvas de nematódeos, no estrato epitelial da pele humana. No Brasil, - $A$. braziliense e o $A$. caninum são os principais agentes etiológicos desta afecção (Nunes et al., 2000). Esta zoonose pode ocorrer nos locais que possuem cães e/ou gatos infectados por ancilostomídeos (Nunes et al., 2000). Porém, esta zoonose se torna mais grave e é mais freqüente em praias e em terrenos arenosos (caixas de areia, campos de futebol, quadras de vôlei), onde as condições ambientais favorecem a evolução destes nematódeos até a sua forma infectante. Em muitos locais, os gatos são as principais fontes de infecção. O hábito que possuem de enterrar suas fezes, e a preferência por fazê-lo em lugares com areia favorecem 0 desenvolvimento das larvas dos ancilostomídeos (Nunes et al., 2000).

A LMV ou toxocaríase ocorre quando o ser humano ingere ovos larvados de $T$. canis ou $T$. cati, onde o estádio larval liberado migra pelas vísceras, SNC e olho. As fontes de infecção para o homem são principalmente os cães e gatos infectados pelos helmintos $T$. canis e $T$. cati, respectivamente. Estes parasitos são altamente prevalentes em todo o mundo em animais domiciliados, semidomiciliados e nos de rua (Guimarães et al., 2005). Em várias cidades do País, numerosa população canina circula livremente pelas ruas e praças públicas, sendo alguns, animais errantes e outros levados pelos proprietários para lazer. Estes animais podem contaminar 0 ambiente com a defecação, contaminando o solo com vários tipos e formas parasitárias potencialmente causadoras de zoonoses (Guimarães et al., 2005; Santarém et al., 1998).

Observando a facilidade da contaminação humana em áreas de lazer torna-se necessário o conhecimento do grau de contaminação desses locais por agentes zoonóticos, para que com estes indicadores, as autoridades sanitárias possam ser alertadas e caso necessário venham a tomar medidas eficazes de controle com a finalidade de diminuir a ocorrência de doenças zoonóticas.

Deste modo, o objetivo deste trabalho foi determinar a ocorrência de ovos de Toxocara spp. e ovos/larvas de Ancylostoma spp. em amostras de areia e de fezes coletadas em áreas de lazer do município de Canoinhas, SC, Brasil. 


\section{MATERIAL E MÉTODOS}

\section{Área de estudo}

O trabalho foi desenvolvido no município de Canoinhas, planalto norte de Santa Catarina, nas coordenadas de $26^{\circ}$ $10^{\prime} \mathrm{S}$ e $50^{\circ}$ 23' $\mathrm{W}$, altitude de $765 \mathrm{~m}$, que tem um clima tipo Cfb (clima temperado constantemente úmido, sem estação seca, com verão fresco, e onde a temperatura média anual se situa entre $15,5^{\circ} \mathrm{C}$ e $17,0^{\circ} \mathrm{C}$ ) (figura 1). No município existem 10 praças e um campo de futebol comunitário. As áreas para a coleta das amostras foram selecionadas aleatoriamente, sendo composta de $50 \%$ das praças $(n=5)$ e $100 \%$ dos campos de futebol comunitário $(n=1)$. Sendo: as Praças Lauro Müller, Osvaldo de Oliveira e Miguel Procopiak, situadas no centro do município; a Praça Bar das Placas, situada no Bairro Tricolin, conhecido no município como ponto turístico por constar no Guiness Book como maior acervo de placas do mundo no Brasil; e o Campo de Futebol de Areia comunitário da empresa Lavrasul S/A, situado no Bairro Industrial I. Nestes locais, entre julho e outubro de 2007 (período que incluiu as estações de inverno e primavera; nas quais as condições ambientais são desfavoráveis e favoráveis respectivamente, à sobrevivência e ao desenvolvimento das formas préparasitárias), eram a cada mês coletas cinco amostras de areia e cinco de fezes. No período foram coletadas 200 amostras, sendo 100 amostras de areia e 100 de fezes das cinco áreas de lazer do município de Canoinhas, SC.

\section{Coleta das Amostras de Areia e Fezes}

As amostras de areia eram coletadas com o auxílio de uma colher, em cinco diferentes locais de cada área de lazer (uma amostra era coletada em cada canto da área com areia, e a última amostra era coletada no centro do local). As amostras de fezes foram coletadas diretamente do solo com o auxílio de sacos plásticos. Todas as amostras eram armazenadas em isopor com gelo e levadas ao laboratório de Parasitologia da UnC, Campus Canoinhas, onde os exames eram realizados no dia da coleta.

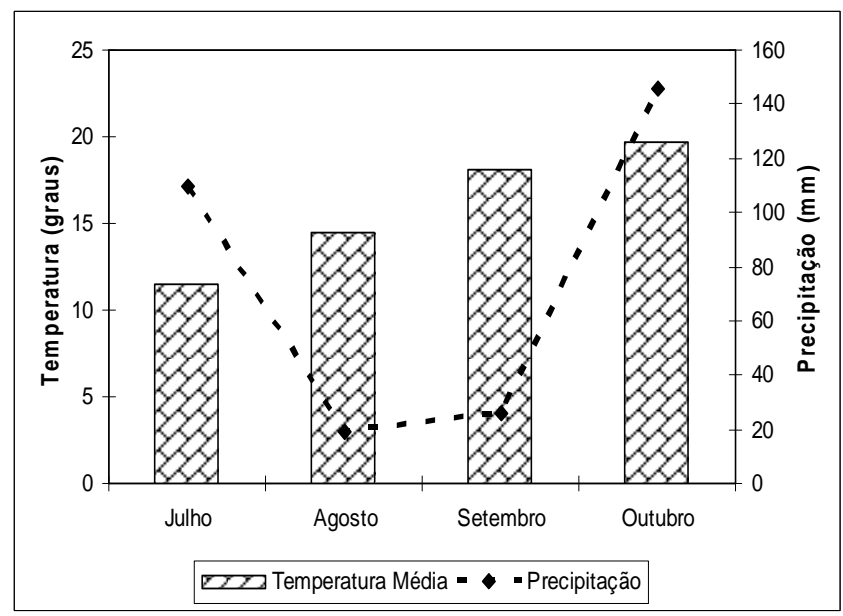

Figura 1 - Temperaturas máxima e mínima e precipitação pluviométrica. Canoinhas/SC, 2007. Fonte: RIGESA

\section{Exames Parasitológicos}

Cada amostra (areia/fezes) coletada foi processada por três métodos de exame parasitológico: sedimentação espontânea, centrífugo-sedimentação e centrífugoflutuação em solução saturada de açúcar (Hoffmann, 1987).

Segundo o método de sedimentação espontânea ou método de Hoffmann, Pons e Janer: foram pesadas $50 \mathrm{~g}$ (para areia) e $5 \mathrm{~g}$ (para fezes), que foram diluídas em 300 $\mathrm{ml}$ de água destilada. Esta solução foi filtrada para um cálice de sedimentação. Depois de passados 30 minutos, o sobrenadante foi decantado, e ao sedimento foram adicionados aproximadamente $200 \mathrm{ml}$ de água destilada. A mistura foi agitada e deixada para sedimentar por mais 30 minutos. Após, foi decantado o líquido sobrenadante e coletadas algumas gotas do sedimento, colocadas entre lâmina e lamínula e examinadas ao microscópio óptico (100X) para pesquisa de formas parasitárias.

Pelo método de centrífugosedimentação ou método de Ritchie: as amostras de areia $(20 \mathrm{~g})$ e de fezes $(2 \mathrm{~g})$ 
foram emulsionadas em $10 \mathrm{ml}$ de água e a seguir filtradas através de uma gaze para um tubo de ensaio. O material foi centrifugado à $2000 \mathrm{rpm}$, durante $2 \mathrm{~min}$. Após desprezado o sobrenadante, 0 sedimento foi ressuspenso em água, centrifugado e decantado novamente $e$ adicionado de $10 \mathrm{ml}$ de solução de formol a $10 \%$ ao sedimento. Após homogeneização foi mantido em repouso por $5 \mathrm{~min}$. Adicionou-se $3 \mathrm{~mL}$ de éter, agitou-se, e foi levado à centrifugação a 1500 rpm por 2 min. $O$ anel formado pelos resíduos, foi removido e foram decantadas as três camadas superiores, e misturado ao sedimento 1 a 2 gotas de lugol. $O$ sedimento foi depositado entre lâmina e lamínula e examinado ao microscópio, sendo lidas várias lâminas de acordo com a quantidade do sedimento (Hoffmann, 1987).

O método da centrífugo-flutuação ou método de Sheather: foi efetuado com a pesagem de um grama de fezes e 10 gramas de areia, que foi dissolvida em solução fisiológica e a seguir filtrada. Tubos de centrífuga foram preenchidos até a metade do volume com esta solução filtrada e o restante foi preenchido com solução saturada de sacarose e a seguir centrifugado a $1500 \mathrm{rpm}$, por 5 minutos. Foram retiradas algumas gotas da camada superficial do líquido centrifugado e examinadas ao microscópio entre lâmina e lamínula (Hoffmann, 1987).

\section{Análise Estatística}

Os resultados obtidos foram analisados estatisticamente pelo Teste $Z$ (teste de proporções) e pelo Teste de Student pelo Programa Estatístico Statgraphycs Centurion com nível de confiança de $95 \%$.

\section{RESULTADOS E DISCUSSÃO}

A presença de ovos de Ancylostoma spp. e ovos de Toxocara spp. em amostras de areia analisadas foi de
$39 \%(39 / 100)$. Nos exames de fezes coletadas nas áreas de lazer foram detectadas 17\% (17/100) de amostras positivas, sendo detectado apenas ovos de Ancylostoma spp.

O percentual de positividade variou com os meses de coleta, de nenhuma amostra de fezes positiva nos meses de julho e agosto, até $100 \%$ de amostras de fezes positivas no mês de outubro. Entretanto, essas diferenças foram significativas estatisticamente apenas entre os meses de setembro e outubro e apenas para as amostras de areia $(P=0,0285)$. Diferente de outros estudos, que detectaram um maior percentual de positividade nas amostras fecais, neste trabalho foram encontradas mais amostras de areia positivas do que de fezes, embora a diferença não tenha sido estatisticamente significativa ( $P=0,1013)$ (Matesco et al., 2006; Nunes et al., 2000).

Das áreas de lazer examinadas, $100 \%$ apresentaram a areia contaminada e $80 \%$ apresentaram fezes contaminadas por Ancylostoma spp e/ou Toxocara spp. Valores similares foram obtidos nos estudos de Corrêa e Moreira (1996), que observaram amostras positivas em 93,3\% das praças examinadas; e de Corrêa et al. (1993), que constataram $95,8 \%$ das praças com fezes contaminadas por ovos de Ancylostoma spp. Em 100\% das áreas de lazer examinadas foi detectado 0 Ancylostoma spp e apenas uma área (80\%) apresentou contaminação com ovos de Toxocara spp.. As praças onde as amostras foram coletadas são locais muito movimentados. Com isso, a permanência de adultos e crianças nesses locais é grande, além da presença de cães e gatos errantes.

$\mathrm{Na}$ Praça Lauro Müller, $35 \%$ das amostras de areia e $15 \%$ das amostras de fezes, apresentaram resultado positivo nos métodos realizados. Já na Praça Miguel Procopiak, que possui uma grande área verde, e é um ponto de encontro de jovens 


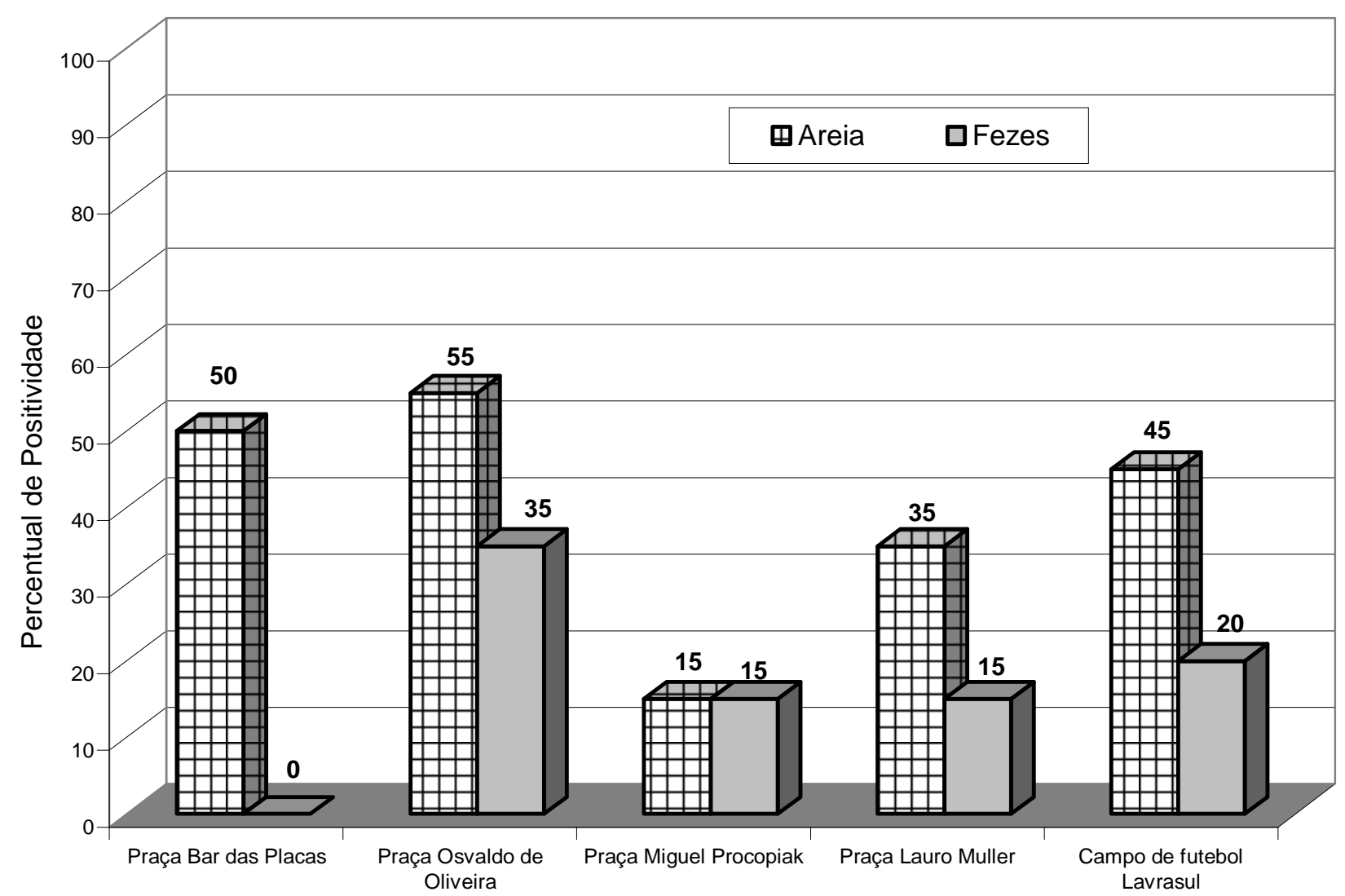

Figura 2 - Percentual de amostras de fezes e areia positivas para Ancylostoma spp. e/ou Toxocara spp. de acordo com a área de lazer do município de Canoinhas/SC analisadas. 2007 ( $n=5$ amostras/mês/área de lazer).

nos finais de semana e de passeio de crianças, pais e seus animais de companhia nos finais de tarde, $15 \%$ das amostras de fezes e $15 \%$ das de areia, apresentaram resultado positivo.

$\mathrm{Na}$ Praça Osvaldo de Oliveira, local de intenso movimento diário, onde se concentram os principais pontos comerciais do município, e grande número de animais perambulam $55 \%$ das amostras de areia e $35 \%$ das amostras de fezes estavam positivas para os agentes pesquisados. $\mathrm{Na}$ Praça do Bar das Placas, $50 \%$ das amostras de areia apresentaram resultado positivo nos métodos utilizados, enquanto as amostras de fezes foram todas negativas. No Campo de Futebol de Areia Lavrasul, onde se concentra grande quantidade de crianças brincando durante o dia, $45 \%$ das amostras de areia e $20 \%$ das amostras de fezes apresentaram resultados positivos nos métodos utilizados. Salienta-se que a área não é cercada, favorecendo o livre acesso de cães e gatos (figura 2).

A areia da praça Miguel Procopiak mostrou-se significativamente menos contaminada que a das praças Bar das Placas $(P=0,039)$ e Osvaldo de Oliveira $(P=0,012)$. Este fato pode ser devido à menor freqüência de pessoas e principalmente de animais na praça Miguel Procopiak. Não houve diferença significativa na contaminação da areia $(P>0,05)$ entre as demais comparações entre as praças.

Para as amostras de fezes, houve diferença significativa entre a contaminação quando comparadas às praças Bar das Placas versus Osvaldo de Oliveira $(\mathrm{P}=0,006)$ e Bar das Placas versus Campo de Futebol de Areia -Lavrasul $(P=0,041)$. 
Essa diferença foi significativa, porque todas as amostras coletadas na praça Bar das Placas foram negativas. Não houve diferença significativa na contaminação das fezes $(P>0,05)$ entre as demais comparações entre as praças.

Das 200 amostras avaliadas (100 de areia e 100 de fezes), $28 \%$ foram positivas em ao menos um dos métodos utilizados. Das 100 amostras de fezes analisadas no período, $17 \%$ foram positivas em no mínimo um dos métodos. Já para as amostras de areia, 39\% das amostras foram positivas em pelo menos um dos métodos. Nas amostras de areia foram observados ovos de Toxocara spp. e Ancylostoma spp.; enquanto que nas amostras de fezes apenas ovos de Ancylostoma spp. foram detectados. Não foram detectadas larvas de Ancylostoma spp nos exames, apenas ovos. A presença de áreas de lazer com amostras de areia positivas para parasitas com potencial zoonótico, indica que há possibilidade de transmissão de zoonoses, principalmente de LMC, pela maior ocorrência de Ancylostoma spp., nas amostras analisadas, principalmente em crianças, que são a principal faixa etária acometida (Labruna et al., 2006; Nunes et al., 2000). Ovos de Toxocara spp., agente da LMV, foram detectados em amostras de areia somente na praça Osvaldo de Oliveira e apenas nos meses de julho e agosto, com $4 \%$ e $8 \%$ de amostras positivas, respectivamente.

Constatou-se que a contaminação das praças públicas por Ancylostoma spp., ocorre com maior freqüência do que a contaminação por Toxocara spp., concordando com os resultados de outros pesquisadores, como Araújo et al. (1999), que também detectaram índices mais elevados de contaminação do solo em praças públicas por Ancylostoma spp.. Este resultado pode ser devido ao fato dos animais não desenvolverem imunidade contra antígenos deste parasito (Boag et al., 2003), permanecendo com infecções residuais na vida adulta e mantendo a contaminação ambiental.

$\mathrm{Na}$ avaliação estatística dos métodos usados quando as amostras eram de areia, houve diferença significativa na detecção de amostras positivas, apenas entre os métodos de sedimentação simples e centrífugo-sedimentação $(P=0,022)$, sendo as demais comparações não significativas (Sedimentação simples versus centrífugo-flutuação - $\mathrm{P}=0,175 \mathrm{e}$ centrífugo-sedimentação versus centrífugoflutuação - $P=0,340)$.

Dos métodos utilizados nas avaliações, o de sedimentação simples apresentou um total de 11 amostras positivas, o método de centrífugosedimentação detectou 24 amostras positivas e no de centrífugo-flutuação, 23 foram positivas (figura 3). Nos exames com amostras de areia, apenas em uma das amostras o resultado foi positivo pelos métodos de sedimentação simples e centrífugo-flutuação. Nos demais exames as amostras deram resultados positivos apenas em um dos três métodos usados. Nos exames com amostras fecais não houve coincidência entre os resultados pelos métodos examinados. As amostras resultavam positivas em apenas um dos três métodos utilizados. Ovos de Ancylostoma spp. foram detectados pelos três métodos, porém os melhores indices de positividade foram nos métodos de centrífugo sedimentação (22\%) e centrífugo flutuação (23\%); quando comparado ao método de sedimentação simples (11\%), o que se justifica em função deste ser um ovo leve. Ovos de Toxocara spp. foram observados apenas no método de centrífugo Sedimentação e apenas em amostras de areia .

As técnicas que apresentaram resultados mais satisfatórios, nas análises das fezes foram as de centrífugosedimentação e centrífugo-flutuação (figura 3), porém na análise estatística os três métodos apresentaram a mesma eficiência em detectar amostras de fezes positivas 


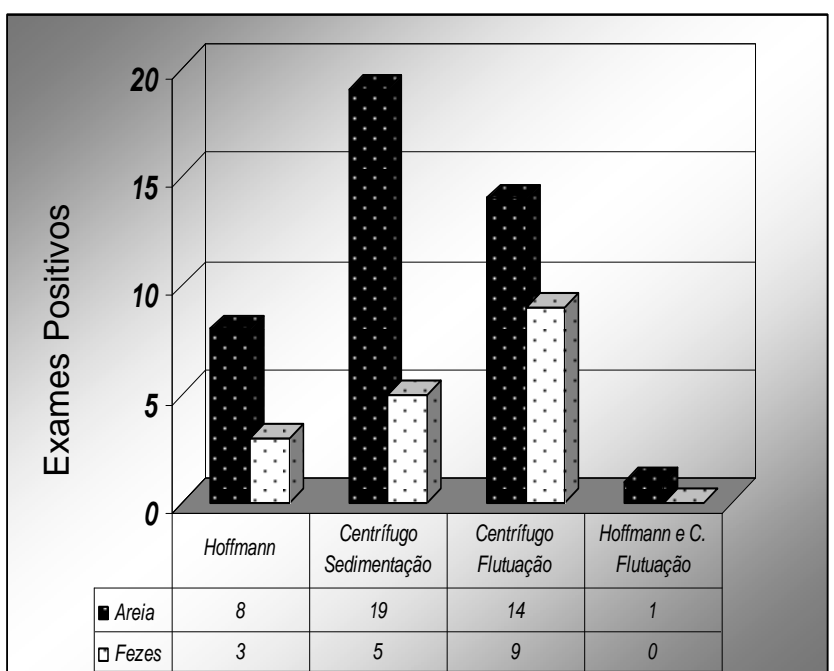

Figura 3 - Número de exames positivos para Ancylostoma spp. e/ou Toxocara spp. de acordo com o método utilizado e material examinado. Canoinhas/SC, 2007.

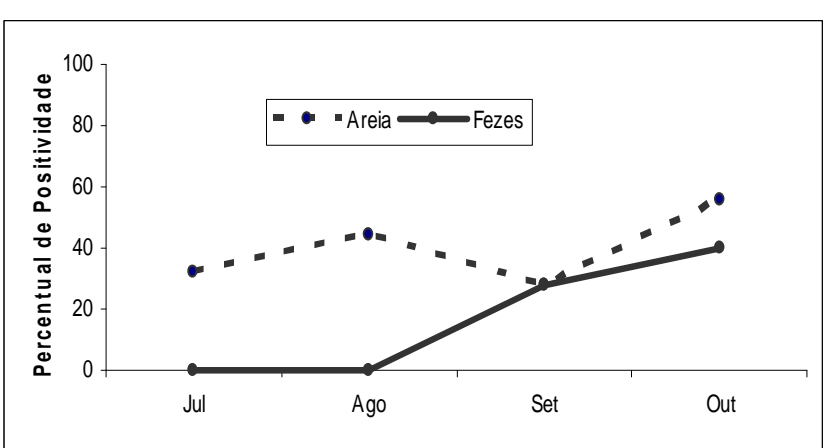

Figura 4 - Percentual de amostras de fezes e areia positivas para Ancylostoma spp. e/ou Toxocara spp. em cinco áreas de lazer do município de Canoinhas/SC, 2007 ( $n=5$ amostras/mês/área de lazer).

$(P>0,05)$. Guimarães et al. (2005) também não obtiveram diferenças significativas ao comparar as técnicas de centrífugo flutuação e de centrífugo flutuação para detecção de ovos em amostras de areia.

As variações climáticas de julho a outubro de 2007 foram significativas (figura 1). Foi observado que, de acordo com o aumento da temperatura do mês de julho até outubro, o percentual geral de amostras de fezes positivas foi aumentando, nos meses de julho e agosto nenhuma amostra de fezes foi positiva, em setembro $28 \%$ foram positivas e em outubro a positividade foi de $40 \%$ (figura 4). A não positividade de fezes nos meses de julho e agosto pode ser reflexo da baixa precipitação pluviométrica, das baixas temperaturas e das geadas no mês de julho, que podem ter favorecido o ressecamento das fezes e inviabilização dos ovos, uma vez que as amostras foram coletadas nos primeiros dias dos respectivos meses. No mês de outubro foi obtido o maior percentual geral de resultados positivos em amostras de areia, bem como em amostras de fezes (figura 4). Isto pode ser reflexo das temperaturas máxima e mínima amenas no mês, bem como da alta precipitação pluviométrica.

\section{CONCLUSÃO}

O estudo revelou que a contaminação das áreas de lazer do município de Canoinhas SC, é preocupante e reforça a necessidade de se implementar medidas efetivas para 0 controle de zoonoses em áreas de lazer, controlando as populações canina e felina presentes nas praças públicas, por meio da apreensão de animais errantes e esterilização cirúrgica dos mesmos, e ainda com a construção de cercas ao redor das áreas de recreação, e cobertura de caixas de areia com lona durante a noite.

\section{REFERÊNCIAS}

ARAUJO, F. R.; CROCCI, A. J.; RODRIGUES, R. G. C.; AVALHAES, J. S.; MIYOSHI, M. I.; SALGADO, F. P.; SILVA, M. A.; PEREIRA, M. L. Contaminação de praças públicas de Campo Grande, Mato Grosso do Sul, Brasil, por ovos de Toxocara e Ancylostoma em fezes de cães. Revista da Sociedade Brasileira de Medicina Tropical, v. 32, n.5, p. 581583, 1999.

BOAG, P.R.; PARSONS, J.C.; PRESIDENTE, P.J.; SPITHILL, T.W.; SEXTON, J.L. Characterisation of humoral immune responses in dogs vaccinated with irradiated Ancylostoma caninum. Veterinary Immunology and Immunopathology, v. 92, p. 8794, 2003.

CORRÊA, G. L. B., GRUNSPAN, E., LAGAGGIO, V. R. A. et al. Pesquisa de ovos e oocistos em fezes de cães e gatos, em praças públicas de Santa Maria e 
sua importância na clínica veterinária e em Saúde Pública. In: CONGRESSO INTERNACIONAL DE MEDICINA VETERINÁRIA EM LÍNGUA PORTUGUESA (6.). Anais... Salvador: Comitê Permanente dos Congressos Internacionais de Medicina Veterinária em Língua Portuguesa, 1993. 447p. p. 336.

CORRÊA, G.L.B.; MOREIRA, W.S. Contaminação do solo por ovos de Ancylostoma spp. em praças públicas, na cidade de Santa Maria, RS, Brasil. Revista da Faculdade de Zootecnia, Veterinária e Agronomia da PUC-RS, v.2/3, p. 18-23, 1996.

GUIMARÃES, A. M.; ALVES, E. G. L.; REZENDE, G. F.; RODRIGUES, M. C. Ovos de Toxocara sp. e larvas de Ancylostoma sp. em praça pública de Lavras, Minas Gerais. Revista de Saúde Pública, São Paulo. v. 39, n. 2, p. 293 - 295. 2005.

HOFFMANN, R. P. Diagnóstico de Parasitismo Veterinário. Porto Alegre: Sulina., p. 39 - 401987.

LABRUNA, M.B.; PENA, H.F.J.; SOUZA, S.L.P.; PINTER, A.; SILVA, J.C.R.; RAGOZO, A.M.A.; CAMARGO, L.M.A.; GENNARI, S.M. Prevalência de endoparasitas em cães da área urbana do município de Monte Negro, Rondônia. Arquivos do Instituto Biológico, São Paulo, v.73, n.2, p.183-193, abr./jun., 2006.

MATESCO, V. C.; MENTZ, M. B.; ROTT, M. B.; SILVEIRA, C.O.. Contaminação sazonal por ovos de helmintos na praia de Ipanema, em Porto Alegre, Rio Grande do Sul, Brasil. Revista de Patologia Tropical, v.35, n.2, p. 135-141, 2006.

NUNES, C. M.; PENA, F. C.; NEGRELLI, G. B.; ANJO, C. G. S.; NAKANO, M. M.; STOBBE, N. S. Ocorrência de larva migrans na areia de áreas de lazer das escolas municipais de ensino infantil, Araçatuba SP, Brasil. Revista Saúde Pública, v. 34, n.6, p. $656-58,2000$.

ROBERTSON, I.D.; IRWIN, P.J.; LYMBERY, A.J.; THOMPSON, R.C.A.. The role of companion animals in the emergence of parasitic zoonoses. International Journal for Parasitology, n. 30, p. 1369-1377, 2000.

SANTARÉM, V. A.; SARTOR, F. I.; BERGAMO, F. M. M. Contaminação, por ovos de Toxocara spp, de parques e praças públicas de Botucatu, São Paulo, Brasil. Revista da Sociedade Brasileira de Medicina Tropical, v. 31, n. 6, p. $529-532.1998$. 